\title{
Changes in Oxidative Stress Biomarkers in the First-Episode Psychosis: A Systematic Review And Meta-Analysis
}

\author{
Joaquim Maria Ferreira Antunes Neto ${ }^{1}$, Ana Maria Nalesso ${ }^{2}$
}

\author{
${ }^{1} \mathrm{PhD}$ in Functional and Molecular Biology, Department of Biochemistry, Institute of Biology, State \\ University of Campinas, São Paulo, Brazil. Professor at the Faculty of Medicine, "Faculdade Municipal \\ Professor Franco Montoro", Mogi Guaçu, São Paulo, Brazil. \\ ${ }^{2}$ Graduating in Psychology, at the "Faculdade Municipal Professor Franco Montoro", Mogi Guaçu, São \\ Paulo, Brazil.
}

\begin{abstract}
The literature has shown that oxidative stress markers can indicate the degree of disease severity in untreated patients with schizophrenia. There is evidence that the excessive production of free radicals or the installation of oxidative stress can be evidenced at the time of the first-episode psychosis (FEP). Were used the Preferred Reporting Items for Systematic Reviews and Meta-analyses Guidelines to conduct a systematic literature search to detect studies comparing oxidative stress markers in FEP patients and healthy controls (HC). Twenty-one studies were obtained for inclusion in this systematic review, comprising an overall sample of 415 FEP and $392 \mathrm{HC}$ for CAT (6 studies), $1293 \mathrm{FEP}$ and $1279 \mathrm{HC}$ for SOD (15 studies), 500 FEP and 529 HC for GPx (9 studies), 218 FEP and 228 HC for GSH (5 estudies), and $814 \mathrm{FEP}$ and $852 \mathrm{HC}$ for lipid peroxidation (12 studies). By analyzing the standardized mean differences, no significant differences were found between FEP and HC groups in any of the antioxidant defense system parameters assessed, despite favoring a decrease in activity of both the antioxidant and non-enzymatic enzyme systems. Lipid peroxidation levels showed a significant increase for the FEP group compared to the HC group ( $p<0.0001$ ). Heterogeneity among studies was high, with $I^{2}$ values $>90 \%$. Sensitivity analyzes were performed to obtain more homogeneous results. Significant differences were obtained for serum/plasma SOD $(\mathrm{p}<0.0001)$ and lipid peroxidation $(\mathrm{p}<0.0001)$ in the FEP group compared to the $\mathrm{HC}$ group, but still containing high heterogeneity. These results suggest that the oxidative stress biomarkers could be used to indicate the degree for the severity of the disease in untreated patients with schizophrenia.
\end{abstract}

Keywords: schizophrenia, first-episode psychosis, biomarker, oxidative stress, meta-analysis.

\section{Introduction}

Schizophrenia is a chronic psychiatric disorder with a neuroprogressive course. Its etiology is multifactorial, with important genetic components and starts in late adolescence or early adulthood and includes symptoms that are positive, negative, affective and cognitive, affecing at least $0.7 \%$ of the population ${ }^{1,2}$. The diagnostic criteria are based on descriptive phenomenology of clinical symptoms and clinical course due to the lack of reliable and specific biomarkers ${ }^{3}$. The neuroprogression theory of schizophrenia postulates that changes in the immune system are accompanied by increased inflammatory markers, tryptophan catabolites and reactive oxygen species (ROS) affecting the growth and function of neuronal circuits since the intra-utero period $^{4}$. There is also an accumulating evidence that oxidative stress plays a role in the etiopathogenesis of mental disorders, particularly schizophrenia and bipolar disorder 5 , which commonly start at early ages ${ }^{6,7}$ with a first-episode psychosis (FEP). Some authors ${ }^{8,9}$, but not all ${ }^{10,11}$, suggest that oxidative injury occurs at the onset of psychosis and they also propose that oxidative stress is a feature of the disease process itself.

Redox state is a regulatory system of posttranslational modifications that controls cellular signalization in response to oxidants and free radicals. Oxidative stress is defined as an imbalance between prooxidants and 
antioxidants, which results in macromolecular damages (lipid peroxidation, protein carbonylation, and DNA oxidation) and in dysregulation of the redox system ${ }^{12}$. Abnormalities induced by oxidative imbalance may contribute to compromised function of neurotransmitters and development of clinically significant consequences in schizophrenia, such as severity of psychopathology and extrapyramidal symptoms ${ }^{13}$. Heightened oxidative stress in schizophrenia may be due to genetic factors leading to decreased capacity to synthesize antioxidant compounds ${ }^{14}$ and/or early developmental insults that can increase generation of reactive oxygen species (ROS) in the brain ${ }^{15}$.

This imbalance may be attributable to a malfunctioning antioxidant system and/or high levels of ROS including superoxide anion radicals $\left(\mathrm{O}_{2}{ }^{\circ}\right)$, hydroxyl radicals $\left(\mathrm{HO}^{*}\right)$, peroxyl radicals $\left(\mathrm{HOO}^{\circ}\right)$, hydrogen peroxide $\left(\mathrm{H}_{2} \mathrm{O}_{2}\right)$, nitric oxide, and reactive nitrogen species (RNS) ${ }^{16} \cdot \mathrm{O}_{2}{ }^{\cdot}$ is central to the formation of ROS; superoxide dismutases (SOD) can transform $\mathrm{O}_{2}{ }^{\cdot}$ into the more stable $\mathrm{H}_{2} \mathrm{O}_{2} \cdot \mathrm{H}_{2} \mathrm{O}_{2}$ can subsequently form highly reactive $\mathrm{HO}^{\circ}$ radicals through the Fenton reaction using $\mathrm{Fe}^{2+}$ as a catalyst. These $\mathrm{HO}^{\circ}$ radicals are among the most cytotoxic and reactive ROS. Conversely, $\mathrm{H}_{2} \mathrm{O}_{2}$ may be decomposed to $\mathrm{H}_{2} \mathrm{O}$ and $\mathrm{O}_{2}$ by catalase (CAT) and peroxidases, as well as by the glutathione system. The glutathione peroxidase (GPx) utilizes reduced glutathione as a hydrogen donor for the removal of peroxides. Thus, it is crucial to maintain the glutathione levels (GSH) at adequate concentrations for normal cell functioning ${ }^{17,18,19}$. However, by modifying lipids, proteins, nucleic acids, and other molecules, excess ROS can be damaging. In particular, increased ROS may lead to lipid peroxidation, which damages cells and organelle membranes ${ }^{13}$. The brain has comparatively greater vulnerability to oxidative damage as a result of the relatively low levels of antioxidants, high levels of polyunsaturated fatty acids and increased need of oxygen ${ }^{20}$. Ciobica and collaborators $^{21}$ showed studies where patients with schizophrenia have generally suggested the presence of a compromised antioxidant system, but this is not always consistent with specific observed parameters, which on the whole, showed evidences of dysregulation.

The diagnosis performed in the FEP has important therapeutic and prognostic implications, considering that patients with mood disorder and those with schizophrenia spectrum disorders are likely to require different medical and psychosocial treatment. Furthermore, FEP patients should be diagnosed and treated urgently, as prolongation of the untreated period results in a poorer prognosis ${ }^{22}$. Thus, the present systematic review and the meta-analyses were derived from studies containing oxidative (lipid peroxidation, including dosages of plasma/serum thiobarbituric acid reactive substances - TBARS and malondialdehydes - MDA), enzymatic antioxidants (CAT, GPx and SOD) and non-enzymatic antioxidant (GSH) parameters in subjects who manifested FEP compared to healthy controls (HC). In this study we tested the hypothesis that the FEP is related to the intensification of the oxidative stress condition. If so, there is the possibility that some prooxidative and antioxidant markers can be used for the early clinical identification of schizophrenia.

\section{Methods}

\section{Search strategy}

A systematic search was conducted on PubMed, Web of Science e Scopus with the last update on April 19, 2021. The search included combined terms to match first-episode psychosis (or "FEP" or "early onset psychosis" or "EOP" or "early onset schizophrenia" or "first-episode schizophrenia" or "early psychosis" or "early schizophrenia") with oxidative stress (or "oxidants" or "antioxidants" or "reactive oxygen species" or "catalase" or "superoxide dismutase" or "glutathione peroxidase" or "glutathione" or "malondialdehyde" or "thiobarbituric acid reactive substances").

The flowchart of study selection is detailed in Figure 1. We included studies showing effects of first-episode psychosis oxidative stress (FEP) and healthy control group (HC) on blood concentration of CAT, SOD, GPx, GSH and lipid peroxidation in adults $>21$ years of age. Studies were excluded if they met any of the following hierarchical exclusion criteria: (1) they were not published as original peer-reviewed articles; (2) they did not include a healthy control group; (3) they did not include a FEP group (to consider this group as 'first episode', all patients included in the study should be experiencing a first episode of psychosis according to DSM-III R, DSM-IV ou PANNS criteria and should be 21 years old at the onset of psychosis; including brief psychotic disorder, psychotic disorder not otherwise specified, schizophreniform disorder, schizophrenia, mania with psychotic symptoms, and depression with psychotic symptoms; none of the reviewed studies included patients with substance-induced psychosis); and (4) they did not report results or data that could reach the mean and standard deviation values of the oxidative stress markers, as well as data on the statistical comparison between patients with FEP and controls. After 
the overall screening, four studies were not included in the meta-analysis due to the presence of conflicting criteria for the characterization of FEP and adolescents in both the experimental and control groups.

Two independent reviewers selected studies and extracted data. Among the 21 studies selected, 06 assessed $\mathrm{CAT}^{10,}{ }^{23-27}, \quad 15 \mathrm{SOD}^{10,22-35}, \quad 9 \mathrm{GPx}^{10,22-24,27,28,31,36,37}, \quad 5 \mathrm{GSH}^{10,20,23,37,38}$ and 12 lipid peroxidation $^{20,22,24,25,27,31,33,35,36,38-40}$. Lipid peroxidation data included experiments analyzing TBARS and malondialdehyde levels. From these studies emerged the data from the meta-analyses seen in Table 2. Mean, standard deviation (SD) and sample number (n) were used for analysis. Standard error (SE) was converted to $\mathrm{SD}$ by the equation $\mathrm{SD}=\mathrm{SE} *(\sqrt{\mathrm{n}})$, if $\mathrm{SD}$ was not provided in the original study. Furthermore, median and interquartile range $(\mathrm{IQR})$ was replaced by median and $\mathrm{SD}(\mathrm{SD}=[\mathrm{IQR} / 1.35])^{42}$. To identify the heterogeneity of the findings, sensitivity analyzes were applied in order to verify whether the type of sample (erythrocyte or serum/plasma) used in the experiments would generate subgroups.

The PEDro scale assessed quality of the studies ${ }^{42}$. Thus, scores on PEDro scale ranged from 0 (very low methodological quality) to 9 (high methodological quality). The quality of the studies was used for qualitative assessment, and it was not an exclusion criteria. Egger's tests were performed to check the risk of publication bias in each of the meta-analyses.

Data extracted from eligible studies seen in Table 1 included: first author; year of the study; age (mean and $\mathrm{SD}$ ); number of FEP patients and HC controls; gender (as number of men/women); diagnosis criteria; tobacco users (as total number); mean and SD for each oxidative stress marker (for both FEP patients and controls); antipsychotic use (yes/no), name of oxidative stress marker included.

\section{Statistical Analysis}

The five meta-analyses were performed using Review Manager Software version versão 5.4.1. The effect size was calculated based on standard mean difference (SMD: difference between the changes within FEP and control groups) and its $95 \%$ CIs were calculated for every individual marker. Based on the known heterogeneity of markers, we expected that the estimates would also vary substantially between studies. Random-effects models assume that the true effect size varies from one study to the next and, accordingly, are more conservative than fixed effect models. Therefore, random effects models were used.

Statistical heterogeneity was assessed through visual inspection of forest plots and using the Q statistic (a magnitude of heterogeneity) and the $I^{2}$ statistic (a measure of the proportion of variance in summary effect size attributable to heterogeneity). It was decided to analyze separately the experiments performed on erythrocytes and plasma/serum samples.

Figure 1. Flowchart of studies selection.

\begin{tabular}{|c|c|c|c|}
\hline Identification & \multicolumn{2}{|c|}{$\begin{array}{l}\text { Records identified through PubMed (63), } \\
\text { Web of Science (73) e Scopus (69) } \\
\text { database searching }(n=205)\end{array}$} & $\begin{array}{l}74 \text { duplicate records } \\
\text { excluded }\end{array}$ \\
\hline \multirow[t]{2}{*}{ Screening } & $\begin{array}{l}\text { Records screened } \\
\text { through database filter } \\
\qquad(n=131)\end{array}$ & \multicolumn{2}{|c|}{$\begin{array}{l}72 \text { records excluded: reviews (54), } \\
\text { animals studies (06), no original studies (12) }\end{array}$} \\
\hline & $\begin{array}{c}\text { Articles selected } \\
\text { through abstract } \\
\text { evaluation }(n=59)\end{array}$ & \multicolumn{2}{|c|}{$\begin{array}{l}31 \text { records excluded: no compatible antioxidant } \\
\text { markers (12), no compatible oxidative markers (09), } \\
\text { only inclusion of chronic/medicated patients (10) }\end{array}$} \\
\hline Eligibility & \begin{tabular}{|c|} 
Full-text articles \\
included in qualitative \\
synthesis $(n=28)$
\end{tabular} & \multicolumn{2}{|c|}{$\begin{array}{c}07 \text { records excluded: doubtful } \\
\text { characterization of FEP (03) and inclusion of } \\
\text { adolescents (04) }\end{array}$} \\
\hline Included & \multicolumn{2}{|c|}{$\begin{array}{l}\text { Studies included in quantitative } \\
\text { synthesis (meta-analysis) }(n=21)\end{array}$} & \\
\hline
\end{tabular}


Table 1. Characteristics of studies included in the meta-analyses $(n=21)$.

\begin{tabular}{|c|c|c|c|c|c|c|c|c|c|c|c|}
\hline \multirow[b]{2}{*}{$\begin{array}{l}\text { First author, } \\
\text { year }\end{array}$} & \multicolumn{6}{|c|}{ Schizophrenia Manifestation (FEP) } & \multicolumn{4}{|c|}{ Healthy Control (HC) } & \multirow[b]{2}{*}{$\begin{array}{l}\text { Markers } \\
\text { included }\end{array}$} \\
\hline & $\begin{array}{l}\text { FEP } \\
(\mathbf{n})\end{array}$ & $\begin{array}{c}\text { Age } \\
(\mathbf{m} \pm \mathrm{SD})\end{array}$ & $\begin{array}{c}\text { Diagnostic } \\
\text { criteria }\end{array}$ & $\begin{array}{l}\text { Gender } \\
(\mathbf{M} / \mathbf{W})\end{array}$ & $\begin{array}{c}\text { Smoke } \\
\mathbf{r} \\
(\mathbf{n}, \%)\end{array}$ & $\begin{array}{l}\text { Drug } \\
\text { naïve }\end{array}$ & $\begin{array}{l}\text { HC } \\
(\mathbf{n})\end{array}$ & $\begin{array}{c}\text { Age } \\
(\mathbf{m} \pm \mathbf{S D})\end{array}$ & $\begin{array}{l}\text { Gender } \\
(\mathbf{h} / \mathbf{m})\end{array}$ & $\begin{array}{l}\text { Smoke } \\
\mathbf{r} \\
(\mathbf{n})\end{array}$ & \\
\hline $\begin{array}{l}\text { Mukherjee et al. } \\
\quad(1996)^{28}\end{array}$ & 14 & $22.82 \pm 4.2$ & DSM-III R & $11 / 3$ & $\mathrm{n} / \mathrm{a}$ & yes & 10 & $28.61 \pm 7.3$ & $6 / 4$ & $\mathrm{n} / \mathrm{a}$ & SOD \\
\hline $\begin{array}{l}\text { Mahadik et al. } \\
\qquad(1998)^{38}\end{array}$ & 26 & $23.3 \pm 4.6$ & DSM-III R & $20 / 6$ & $\mathrm{n} / \mathrm{a}$ & yes & 10 & $27.9 \pm 8.3$ & $6 / 4$ & $\mathrm{n} / \mathrm{a}$ & TBARS \\
\hline $\begin{array}{l}\text { Kartalci et al. } \\
\qquad(2011)^{20}\end{array}$ & 28 & $30.3 \pm 7.6$ & DSM-IV & $15 / 13$ & 13 & yes & 20 & $29.5 \pm 9.7$ & $10 / 10$ & 9 & MDA, GSH \\
\hline $\begin{array}{l}\text { Raffa et al. } \\
(2011)^{23}\end{array}$ & 23 & $29.3 \pm 7.5$ & DSM-IV & $20 / 3$ & 13 & yes & 45 & $29.6 \pm 6.2$ & $36 / 9$ & 11 & $\begin{array}{l}\text { SOD, GPx, } \\
\text { CAT, GSH }\end{array}$ \\
\hline $\begin{array}{l}\text { Khan et al. } \\
(2012)^{39}\end{array}$ & 22 & $22.40 \pm 4.8$ & DSM-IV & $18 / 4$ & $\mathrm{n} / \mathrm{a}$ & yes & 16 & $24 \pm 5.6$ & $14 / 2$ & $\mathrm{n} / \mathrm{a}$ & TBARS \\
\hline $\begin{array}{c}\text { Martínez- } \\
\text { Cengotitabengoa } \\
\text { et al. }(2012)^{10}\end{array}$ & 28 & $27.68 \pm 9.18$ & DSM-IV & $21 / 7$ & $\mathrm{n} / \mathrm{a}$ & yes & 28 & $27.95 \pm 7.50$ & $21 / 7$ & $\mathrm{n} / \mathrm{a}$ & $\begin{array}{l}\text { CAT, SOD, } \\
\text { GPx, GSH }\end{array}$ \\
\hline $\begin{array}{l}\text { Wu et al. } \\
(2012)^{29}\end{array}$ & 78 & $29.4 \pm 9.1$ & DSM-IV & $42 / 36$ & 27 & yes & 100 & $29.9 \pm 10.9$ & $65 / 35$ & 32 & SOD \\
\hline $\begin{array}{l}\text { Ozornina et al. } \\
\qquad(2013)^{24}\end{array}$ & 66 & $24.5 \pm 05$ & $\begin{array}{l}\text { ICD-10 } \\
\text { PANSS }\end{array}$ & $34 / 22$ & $\mathrm{n} / \mathrm{a}$ & yes & 20 & $24.5 \pm 05$ & $11 / 9$ & $\mathrm{n} / \mathrm{a}$ & $\begin{array}{c}\text { SOD, CAT, } \\
\text { GPx, TBARS }\end{array}$ \\
\hline $\begin{array}{l}\text { Noto et al. } \\
(2015)^{40}\end{array}$ & 51 & $25.4 \pm 7.5$ & DSM-IV & $36 / 15$ & 10 & yes & 61 & $26 \pm 7.3$ & $32 / 29$ & 0 & $\mathrm{LOOH}$ \\
\hline $\begin{array}{l}\text { Sarandol et al. } \\
\quad(2015)^{22}\end{array}$ & 26 & $25.6 \pm 7$ & DSM-IV & $10 / 16$ & 19 & yes & 25 & $23.5 \pm 9.2$ & $10 / 15$ & 19 & $\begin{array}{l}\text { MDA, SOD, } \\
\text { GPx }\end{array}$ \\
\hline $\begin{array}{l}\text { Camkurt et al. } \\
(2016)^{25}\end{array}$ & 50 & $36.4 \pm 12.4$ & DSM-IV & $16 / 34$ & $\mathrm{n} / \mathrm{a}$ & yes & 50 & $33.84 \pm 10.7$ & $22 / 28$ & $\mathrm{n} / \mathrm{a}$ & $\begin{array}{l}\text { MDA, CAT, } \\
\text { SOD }\end{array}$ \\
\hline $\begin{array}{l}\text { Bai et al. } \\
(2018)^{30}\end{array}$ & 40 & $27.13 \pm 1.06$ & PANSS & $16 / 24$ & $\mathrm{n} / \mathrm{a}$ & yes & 80 & $\begin{array}{c}27.48 \pm \\
0.069\end{array}$ & $44 / 36$ & $\mathrm{n} / \mathrm{a}$ & $\begin{array}{l}\text { GPx, SOD, } \\
\text { MDA }\end{array}$ \\
\hline $\begin{array}{l}\text { Jordan et al. } \\
\qquad(2018)^{31}\end{array}$ & 22 & $30 \pm 2.62$ & PANSS & $13 / 9$ & 16 & yes & 43 & $35 \pm 4.45$ & $26 / 17$ & 15 & $\begin{array}{l}\text { MDA, SOD, } \\
\text { TSG }\end{array}$ \\
\hline $\begin{array}{l}\text { Langbein et al. } \\
\qquad(2017)^{36}\end{array}$ & 27 & $25.3 \pm 3.52$ & PANSS & $13 / 14$ & 11 & yes & 31 & $25.12 \pm 4.68$ & $18 / 13$ & 12 & $\begin{array}{l}\text { TBARS, } \\
\text { GSHt, GPx, }\end{array}$ \\
\hline $\begin{array}{l}\text { Miljević et al. } \\
\qquad(2018)^{26}\end{array}$ & 19 & $24.2 \pm 1.4$ & ICD-10 & $\mathrm{n} / \mathrm{a}$ & 13 & yes & 20 & $27 \pm 2$ & n.e. & 10 & $\begin{array}{l}\text { SOD, CAT, } \\
\text { GPx }\end{array}$ \\
\hline $\begin{array}{l}\text { Reyes-Madrigal et } \\
\text { al. }(2019)^{37}\end{array}$ & 10 & $22.30 \pm 4.42$ & DSM-IV & $5 / 5$ & 1 & yes & 9 & $22.56 \pm 2.35$ & $5 / 4$ & 0 & GSH \\
\hline $\begin{array}{l}\text { Lang et al. } \\
(2020)^{32}\end{array}$ & 166 & $25.8 \pm 9.1$ & DSM-IV & $98 / 67$ & 40 & yes & 133 & $27.7 \pm 7.6$ & $70 / 63$ & 39 & SOD \\
\hline $\begin{array}{l}\text { Wang et al. } \\
(2020)^{34}\end{array}$ & 163 & $26 \pm 9.2$ & DSM-IV & $96 / 67$ & 38 & yes & 133 & $27.75 \pm 7.5$ & $70 / 63$ & 39 & SOD \\
\hline $\begin{array}{l}\text { Xiu et al. } \\
(2020)^{33}\end{array}$ & 327 & $26.9 \pm 9.4$ & DSM-IV & $160 / 118$ & $28.3 \%$ & yes & 391 & $27.7 \pm 7.4$ & $208 / 18$ & $33 \%$ & SOD, MDA \\
\hline $\begin{array}{l}\text { Zhu et al. } \\
(2020)^{27}\end{array}$ & 119 & $29.07 \pm 7.71$ & DSM-IV & $76 / 43$ & 12 & yes & 135 & $29.38 \pm 7.21$ & $80 / 55$ & 9 & $\begin{array}{l}\text { SOD, GPx, } \\
\text { CAT, MDA }\end{array}$ \\
\hline $\begin{array}{l}\text { Reyazuddin et al. } \\
\qquad(2021)^{35}\end{array}$ & 50 & $28.33 \pm 10.9$ & $\begin{array}{l}\text { ICD-10 } \\
\text { PANSS }\end{array}$ & $10 / 40$ & $\mathrm{n} / \mathrm{a}$ & yes & 50 & $\begin{array}{c}29.54 \pm \\
11.90\end{array}$ & $12 / 38$ & $\mathrm{n} / \mathrm{a}$ & $\begin{array}{l}\text { GPx, SOD, } \\
\text { MDA }\end{array}$ \\
\hline
\end{tabular}

Abbreviations: $\mathrm{FEP}=$ first_episode psychosis; $\mathrm{HC}=$ healthy control; $\mathrm{n} / \mathrm{a}=$ not avaliable; $\mathrm{n}=$ number; $\mathrm{m}=\mathrm{mean} ; \mathrm{SD}=\mathrm{standard}$ desviation; $\mathrm{M}=$ men; $\mathrm{W}=$ women; $\mathrm{CAT}=$ catalase; $\mathrm{SOD}=$ superoxide dismutase $; \mathrm{GPx}=$ glutathione peroxidase; GSH = glutathione; TBARS = thiobarbituric acid reactive substances; $\mathrm{MDA}=$ malondialdehydes; $\mathrm{LOOH}=$ lipid hydroperoxides; $\mathrm{TSG}=$ total shulfhydryl groups; GSHt = total glutathione; DSM = Diagnostic and Statistical Manual of Mental Disorders; PANSS = Pretrained Audio Neural Networks; ICD-10 = International Classification of Diseases, Tenth Revision. 


\section{Results}

Table 2 presents the selected markers for the meta-analyses of the enzymatic $\left(\mathrm{CAT}^{10,23-27}, \mathrm{SOD}^{10,22-35}\right.$, and $\mathrm{GPx}^{10,22-24,27,28,31,36,37}$ ) and non-enzymatic $\left(\mathrm{GSH}^{10,20,23,37,38}\right)$ antioxidant systems, such as the oxidative attack marker, the lipid peroxidation ${ }^{20,22,24,25,27,31,33,35,36,38-40}$.

Table 2. Effect size between FEP and HC groups for markers evaluated in meta-analyses.

\begin{tabular}{|c|c|c|c|c|c|c|c|c|c|c|}
\hline \multicolumn{4}{|c|}{ Random Model } & \multicolumn{3}{|c|}{ Effect Size } & \multicolumn{4}{|c|}{ Heterogeneity } \\
\hline Marker & $\begin{array}{l}\text { Studies } \\
\text { (n) }\end{array}$ & $\begin{array}{c}\text { FEP } \\
\text { (n) }\end{array}$ & $\begin{array}{c}\mathbf{H C} \\
\text { (n) }\end{array}$ & $\begin{array}{c}\text { SMD } \\
(95 \% \mathrm{CI})\end{array}$ & $\mathbf{Z}$ & $\mathbf{P}$ & $\mathbf{Q}$ & $\mathbf{P}(\mathbf{Q})$ & $I^{2}$ & $\mathrm{Tau}^{2}$ \\
\hline CAT & 06 & 415 & 392 & $\begin{array}{c}-0.42 \\
(-0.91,0.07)\end{array}$ & 1.69 & 0.09 & 59.82 & 0.00001 & $90 \%$ & 0.37 \\
\hline SOD & 15 & 1293 & 1279 & $\begin{array}{c}-0.30 \\
(-0.90,0.30)\end{array}$ & 0.99 & 0.32 & 630.88 & 0.00001 & $98 \%$ & 1.42 \\
\hline GPx & 9 & 500 & 529 & $\begin{array}{c}-1.39 \\
(-2.64,-0.15)\end{array}$ & 2.19 & 0.03 & 563.51 & 0.00001 & $98 \%$ & 3.94 \\
\hline GSH & 5 & 218 & 228 & $\begin{array}{c}-0.91 \\
(-2.57,0.76)\end{array}$ & 1.07 & 0.29 & 248.88 & 0.00001 & $98 \%$ & 4.24 \\
\hline LP & 12 & 814 & 852 & $\begin{array}{c}+1.75 \\
(0.93,2.57)\end{array}$ & 4.18 & $<0.0001$ & 483.32 & 0.00001 & $98 \%$ & 1.99 \\
\hline
\end{tabular}

Abbreviations: $\mathrm{FEP}=$ first_episode psychosis; $\mathrm{HC}=$ healthy control; $\mathrm{n}=$ number; $\mathrm{SMD}=$ standardized mean difference; $95 \% \mathrm{CI}=95$ confidence interval considering mean value (maximum value, minimum value); CAT = catalase; SOD = superoxide dismutase; GPx glutathione peroxidase; GSH = glutathione; $\mathrm{LP}=$ lipid peroxidation.

A total of 5 markers were addressed by 6 or more studies: CAT, SOD, GPx, GSH, and lipid peroxidation. Therefore, 5 meta-analyses were performed assessing these oxidative markers from 21 studies, as shown in Table 2, comprising an overall sample of 415 FEP and 392 HC for CAT (6 studies), 1293 FEP and 1279 HC for SOD (15 studies), 500 FEP and 529 HC for GPx (9 studies), 218 FEP and 228 HC for GSH (5 estudies), and $814 \mathrm{FEP}$ and $852 \mathrm{HC}$ for lipid peroxidation (12 studies). No significant differences were found between FEP and HC groups in any of the antioxidant defense system parameters (CAT, SOD, GPx, and GSH) assessed, despite favoring a decrease in activity of both the antioxidant and non-enzymatic enzyme systems (seen in the mean values for SMD in Table 2). Lipid peroxidation levels showed a significant increase for the FEP group compared to the HC group $(\mathrm{p}<0.0001)$. Heterogeneity among studies was high, with $I^{2}$ values $>90 \%$.

Sensitivity analyzes were performed to obtain more homogeneous results with this new form of analysis, by excluding studies identified as sources of heterogeneity. In the present study, we chose to analyze only data extracted from experiments performed with erythrocyte samples for CAT $^{10,24,26,27}$ (Figure 2A), $\mathrm{GPx}^{10,24,25,27,36}$ (Figure 2C) and $\mathrm{GSH}^{10,24}$ (Figure 3) and plasma or serum for SOD ${ }^{10,28,30-35}$ (Figure 2B) and lipid peroxidation ${ }^{20,22,24,25,27,31,35,36,38-40}$ (Figure 4).

Figure 2. Forest plot of main results of the erythrocyte (CAT, GPx) and plasma/serum (SOD) antioxidant enzymatic system comparing FEP and HC groups.

\section{A) Erythrocyte CAT}




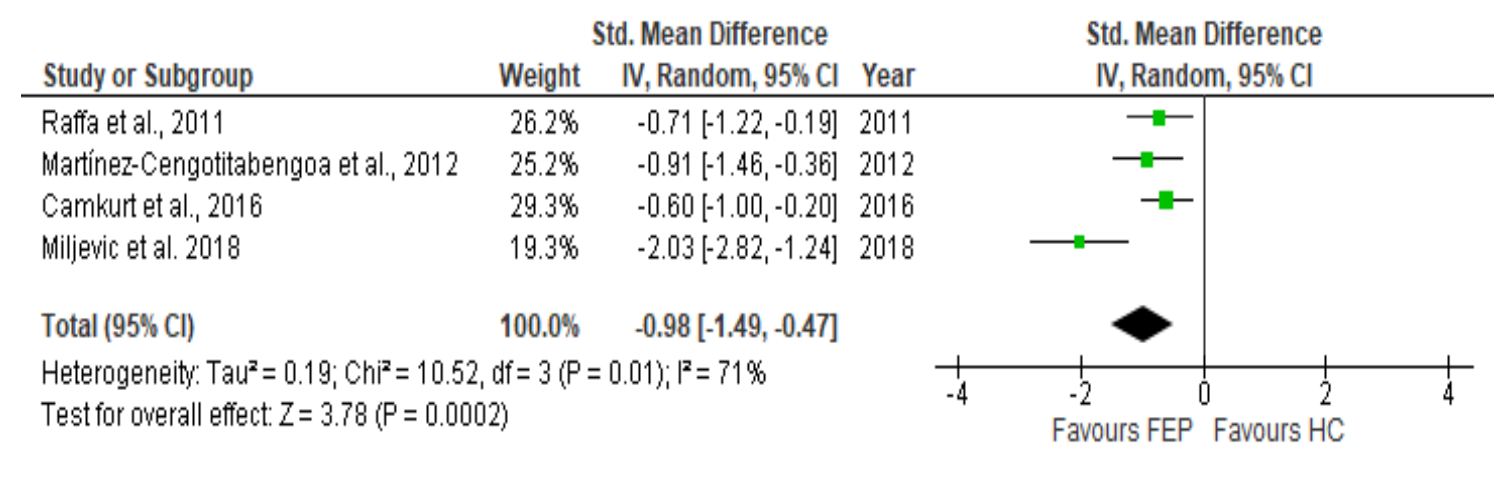

B) Plasma/Serum SOD

\begin{tabular}{|c|c|c|c|c|c|}
\hline Study or Subgroup & \multicolumn{3}{|c|}{ Std. Mean Difference } & \multicolumn{2}{|c|}{$\begin{array}{l}\text { Std. Mean Difference } \\
\text { IV, Random, } 95 \% \mathrm{Cl}\end{array}$} \\
\hline Wu et al., 2012 & $12.2 \%$ & $0.50[0.06,0.94]$ & 2012 & & 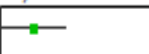 \\
\hline Martínez-Cengotitabengoa et al., 2012 & $11.7 \%$ & $0.29[-0.24,0.81]$ & 2012 & & \\
\hline Bai et al., 2018 & $12.3 \%$ & $1.52[1.10,1.95]$ & 2018 & & - \\
\hline Jordan et al., 2018 & $10.1 \%$ & $3.02[2.27,3.76]$ & 2018 & & \\
\hline Xiu et al., 2020 & $13.7 \%$ & $0.88[0.73,1.04]$ & 2020 & & + \\
\hline Zhu et al., 2020 & $13.3 \%$ & $-0.03[-0.28,0.22]$ & 2020 & & \\
\hline Lang et al., 2020 & $13.4 \%$ & $0.50[0.27,0.73]$ & 2020 & & $\rightarrow$ \\
\hline Wang et al., 2021 & $13.3 \%$ & $1.48[1.22,1.74]$ & 2021 & & $\rightarrow$ \\
\hline Total $(95 \% \mathrm{Cl})$ & $100.0 \%$ & $0.97[0.52,1.42]$ & & & \\
\hline $\begin{array}{l}\text { Heterogeneity: } \text { Tau }^{2}=0.38 ; \mathrm{Chi}^{2}=128.2 \\
\text { Test for overall effect: } Z=4.21 \text { (P } \leq 0.00\end{array}$ & $\begin{array}{l}5, d f=7(f \\
1)\end{array}$ & $0.00001)\left._{i}\right|^{2}=95 \%$ & & $\begin{array}{lll}-2 & -1 & 0 \\
\text { Favours FEP }\end{array}$ & $\begin{array}{cr}1 & 1 \\
\text { Favours } \mathrm{HC}\end{array}$ \\
\hline
\end{tabular}

\section{C) Erythrocyte GPx}

\begin{tabular}{|c|c|c|c|c|c|}
\hline Study or Subgroup & \multicolumn{3}{|c|}{ Std. Mean Difference } & \multicolumn{2}{|c|}{$\begin{array}{l}\text { Std. Mean Difference } \\
\text { IV, Random, } 95 \% \mathrm{Cl}\end{array}$} \\
\hline Raffa et al., 2011 & $20.2 \%$ & $1.19[0.65,1.73]$ & 2011 & & $\because-$ \\
\hline Martínez-Cengotitabengoa et al., 2012 & $20.1 \%$ & $-1.38[-1.96,-0.79]$ & 2012 & - & \\
\hline Ozornina et al., 2013 & $20.2 \%$ & $-1.21[-1.75,-0.68]$ & 2013 & $\rightarrow-$ & \\
\hline Miljevic et al., 2018 & $19.8 \%$ & $-1.86[-2.63,-1.10]$ & 2018 & $\rightarrow-$ & \\
\hline Reyazuddin et al., 2021 & $19.8 \%$ & $-4.64[-5.40,-3.87]$ & 2021 & $\rightarrow-$ & \\
\hline Total (95\% Cl) & $100.0 \%$ & $-1.57[-3.30,0.17]$ & & & \\
\hline \multicolumn{4}{|c|}{$\begin{array}{l}\text { Heterogeneity: } \text { Tau }^{2}=3.81 ; \mathrm{Chi}^{2}=155.32, \mathrm{df}=4(\mathrm{P}<0.00001) ;\left.\right|^{2}=97 \% \\
\text { Test for overall effect: } Z=1.77(\mathrm{P}=0.08)\end{array}$} & $\begin{array}{ll}-4 & -2 \\
\text { Favours FEP }\end{array}$ & $\begin{array}{cc}1 & 1 \\
2 & 4 \\
\text { Favours } \mathrm{HC}\end{array}$ \\
\hline
\end{tabular}

Abbreviations: FEP = first-episode psychosis; $\mathrm{HC}=$ healthy control; $\mathrm{CAT}=$ catalase $; \mathrm{SOD}=$ superoxide dismutase $;$ GPx = glutathione peroxidase. 
Figure 3. Forest plot of main results of the erythrocyte non-enzymatic antioxidant marker (GSH) comparing FEP and HC groups.

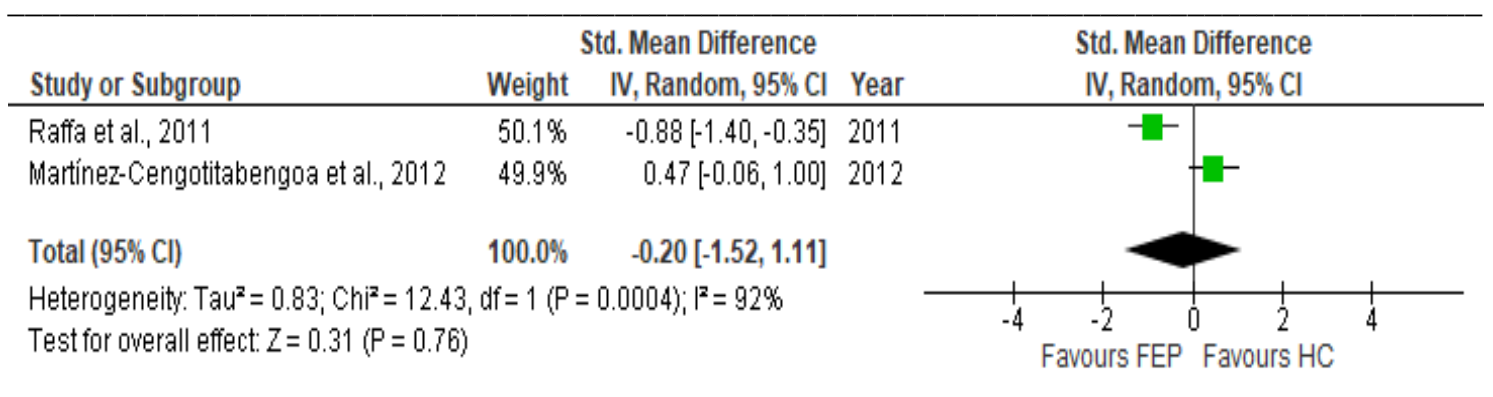

Abbreviations: FEP = first-episode psychosis; $\mathrm{HC}=$ healthy control; $\mathrm{GSH}=$ glutathione.

Figure 4. Forest plot of main results of plasma/serum lipid peroxidation marker comparing FEP and HC groups.

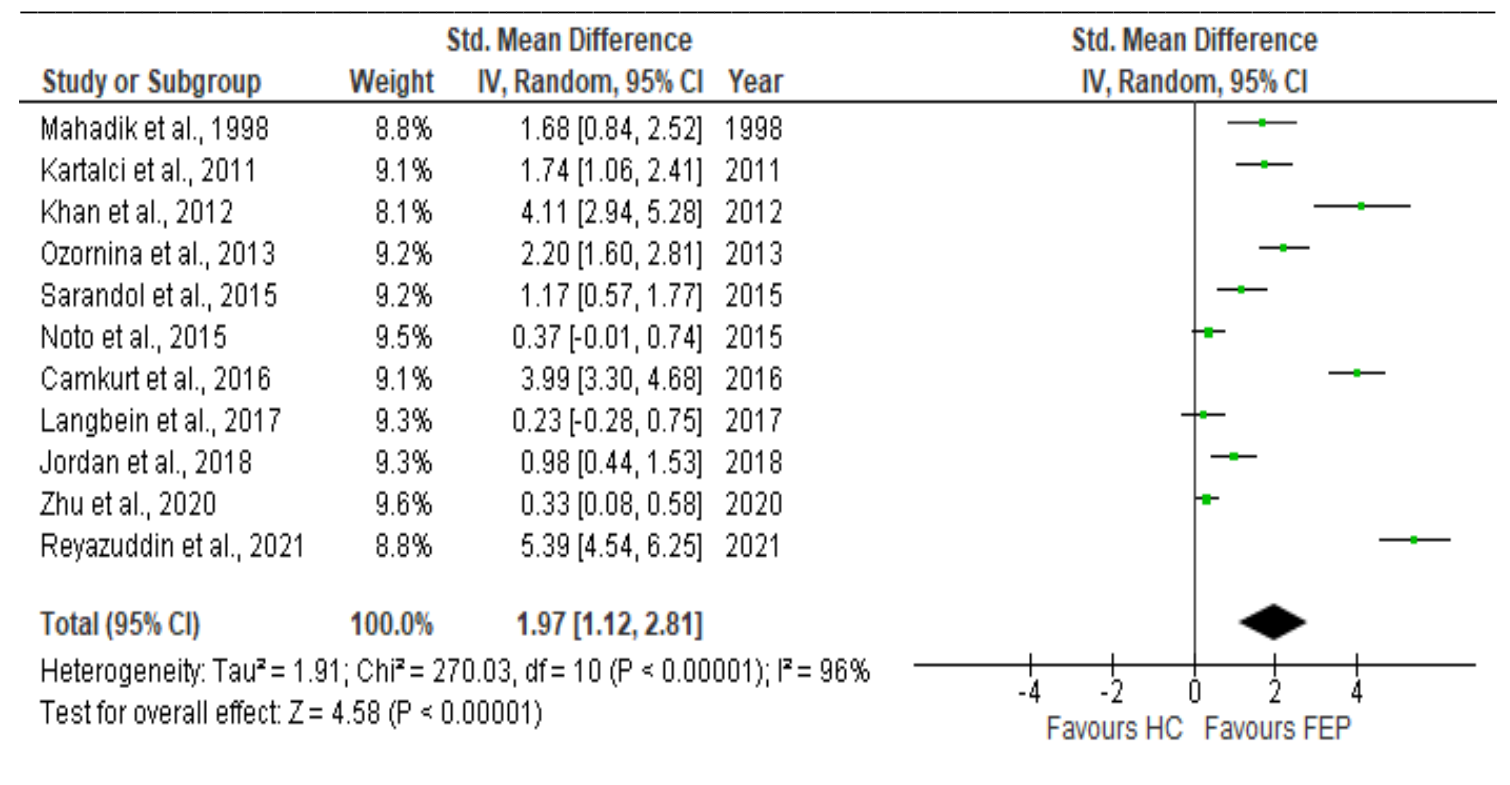

Abbreviations: $\mathrm{FEP}=$ first-episode psychosis; $\mathrm{HC}=$ healthy control.

The proposal above was to understand in these two subgroups (erythrocytes and serum/plasma) which is the best strategy to understand the effect of oxidative stress in FEP patients. Therefore, another 5 meta-analyses were performed assessing these oxidative markers from 21 studies, comprising an overall sample of 120 FEP patients and $143 \mathrm{HC}$ for $\mathrm{CAT}^{10,24,26,27}$ (4 studies), $943 \mathrm{FEP}$ and $970 \mathrm{HC}$ for SOD ${ }^{10,28,30-35}$ (8 studies), $186 \mathrm{FEP}$ and $163 \mathrm{HC}$ for GPx ${ }^{10,24,25,27,36}$ (5 studies), 51 FEP and $73 \mathrm{HC}$ for $\mathrm{GSH}^{10,24}$ (2 estudies), and 487 FEP and $461 \mathrm{HC}$ for lipid peroxidation ${ }^{20,22,24,25,27,31,35,36,38-40}$ (11 studies). We analyzed for each marker both types of samples, erythrocyte and plasma/serum, and the forest plots showed the most significant values obtained. Significant differences were obtained for serum/plasma SOD ( $p<0.0001)$ and lipid peroxidation ( $\mathrm{p}<0.0001)$, but still containing high heterogeneity $(\mathrm{p}<0.0001)$. Interestingly, there was an increase in SOD activity compared to the other two antioxidant enzymes, erythrocyte CAT and GPx, which showed a decrease in activity. The increased levels of serum/plasma lipid peroxidation support the hypothesis of oxidative stress in the etiology of FEP.

\section{Discussion}

A variety of methods have previously been employed to assess oxidative stress within schizophrenia. A large number of studies have assessed peripheral biomarkers of oxidative stress such as antioxidant levels ${ }^{43}$. In the present study, meta-analyses of 5 antioxidants markers to FEP and HC groups did not show any significant results, with great heterogeneity among studies, and even opposite effects found for some markers in different studies, considering the results in Table 2. These contradictory findings may be due, to some extent, to large differences between samples, including different evaluations and analytical methods and clinical factors that can alter oxidative stress levels parameters. Also, most of the studies provided 
insufficient information or not control for confounding factors such as body mass index, gender-based diferences, disease development stage and substance or tobacco use ${ }^{44,45}$. The family members' perception of what would be the FEP can also be a primary limiting factor.

We replicated the findings for antioxidant enzyme markers considering only one sample type (erythrocyte or plasma/serum) as experimental and sample procedures could affect an accurate analysis of antioxidant events, as shown in Figures 2A (CAT), 2B (SOD) and 2C (GPx). Despite the evidence of favoring the decrease of the antioxidant defense systems (enzymatic and non-enzymatic) in FEP, according to Table 2, in experiments with both erythrocyte and plasma/serum samples, a new condition emerged for analysis in plasma/serum in SOD (Figure 2B) when we found that there was an increase in the activity of this enzyme favoring the FEP group in relation to the HC group. The activity values for CAT and GPx were also closer to significance in studies involving only erythrocyte analysis (Figures $2 \mathrm{~A}$ and $2 \mathrm{~B}$, respectively). The SOD metabolizes $\mathrm{O}_{2}{ }^{\cdot}$ into $\mathrm{H}_{2} \mathrm{O}_{2}$ in an attempt to attenuate the formation of more offensive radical species, such as the $\mathrm{HO}^{\circ}$ radicals. This may be the reason for the increase in SOD activity with the consequent decrease in CAT and GPx activities, which denotes an imbalance in the antioxidant enzymatic defense system and a modulation condition on the initial state of events that trigger $\mathrm{FEP}^{30}$.

Although not significant, the decrease in GSH levels, shown both in Table 2 and Figure 3, can change the redox condition of antioxidant enzymes ${ }^{46}$. Perhaps a small change in molecular markers, even without statistical significance, could alter the dynamics of antioxidant defense events and emerge as triggers in the process of oxidative stress in FEP. Finally, the oxidative stress condition is notorious when there is a significant increase in lipid peroxidation levels only in plasma/serum samples. Our meta-analyses collaborate with the finding that the oxidant and antioxidant defense systems indicators have shown that antioxidant defense system defects reflect early pathophysiologic changes in schizophrenics ${ }^{44}$. But it should be noted that even performing sensitivity analyzes for all markers, it was not possible to reduce the heterogeneity of the results obtained. There is a need for more studies that only isolate the same experimental conditions, with a careful selection of subjects reported as FEP.

Estimating levels of reactive oxidative products provides a very useful strategy to determine the impact of oxidative stress. Lipid peroxidation is often assayed by measuring thiobarbituric acid reactive substances (TBARS). The end products of lipid peroxidation, such as MDA assessment, have been very widely used indexes of oxidative stress in clinical studies ${ }^{47,48,49}$. Also, elevated levels of MDA have been shown in plasma, erythrocytes, leucocytes and platelets of patients with schizophrenia ${ }^{50}$. The brain has comparatively greater vulnerability to oxidative damage as a result of the relatively low levels of antioxidants, high levels of polyunsaturated fatty acids and increased need of oxygen ${ }^{51,52}$. There is increasing evidence suggesting the involvement of free radical-mediated neuronal dysfunction in schizophrenia ${ }^{53,54,55,56,57}$, which might be the molecular key to the manifestation of FEP according to the results of the meta-analyses presented.

\section{Conclusions}

The meta-analyses showed a favoring of the decreases in CAT and GPx activities in erythrocyte samples, as well as a significant increase in SOD activity in serum/plasma in the FEP group compared to the HC group, it should be considered that there is a modulation strategy to contain the installation of the oxidative stress condition. This condition can be an initial event that signals the triggering of FEP. Evidence allows us to consider that the operating system activation key in the pathophysiology of schizophrenia has some level of relationship with the imbalance between pro-oxidant and antioxidant molecules within cells and tissues. Cellular damage, seen here by lipid peroxidation, in the intervention of this operating system, appears to be present in the early stages of schizophrenia and other psychoses. Thus, an initial diagnostic strategy by analyzing the oxidative stress markers analyzed here can aid clinical decision-making and help stop the progression and severity of the disorder.

\section{Acknowledgment}

Ana Maria Nalesso is grateful for her participation in the Institutional Scientific Initiation Scholarship Program at "Faculdade Municipal Professor Franco Montoro", Mogi Guaçu, São Paulo, Brazil.

\section{References}


1. Boll KM, Noto C, Bonifácio KL, Bostolasci CC, Gadelha A, Bressan RA, Barbosa DS, Maes M, Moreira EG. Oxidative and nitrosative stress biomarkers in chronic schizophrenia. Psychiatry Research. 2017; 253: 43-48. http://dx.doi.org/10.1016/j.psychres.2017.03.038

2. Garcia-Alvarez L, Garcia-Portilla MP, Gonzalez-Blanco L, Saiz-Martinez PA, Fuente-Tomas L, Menedez-Miranda I, Iglesias C, Bobes J. Differential blood-based biomarkers of psychopathological dimensions of schizophrenia. Rev Psiquiatr Salud Ment (Barc.). 2016; 9:219-227. https://doi.org/10.1016/j.rpsmen.2016.04.008

3. Meana JJ, Mollinedo-Gajate I. Biomarkers in psychiatry: between myth and clinical reality. Rev Psiquiatr Salud Ment. 2017; 10:183-184. https://doi.org/10.1016/j.rpsm.2017.04.003

4. Chan, RC, Di, X, McAlonan, GM, Gong, QY. Brain anatomical abnormalities in high-risk individuals, first-episode, and chronic schizophrenia: an activation likelihood estimationmetaanalysis of illness progression. Schizophr. Bull. 2011; 37:177e188. https://doi.org/10.1093/schbul/sbp073

5. Ng F, Berk M, Dean O, Bush AI. Oxidative stress in psychiatric disorders: evidence base and therapeutic implications. Int. J. Neuropsychopharmacol. 2008; 11: 851-876. https://doi.org/10.1017/s1461145707008401

6. Kaur T, Cadenhead KS. Treatment implications of the schizophrenia prodrome. Curr. Top. Behav. Neurosci. 2010; 4: 97-121. https://doi.org/10.1007/7854_2010_56

7. Lish JD, Dime-Meenan S, Whybrow PC, Price RA, Hirschfeld RM. The National Depressive and Manicdepressive Association (DMDA) survey of bipolar members. J. Affect. Disord. 1994; 31: 281294. https://doi.org/10.1016/0165-0327(94)90104-X

8. Şimşek Ş, Gençoğlan S, Yüksel T, Kaplan İ, Alaca R, Aktaş H. Oxidative stress and DNA damage in untreated first-episode psychosis in adolescents. Neuropsychobiology. 2016; 73: 92-97. https://doi.org/10.1159/000444488

9. Yao JK, Reddy RD, van Kammen DP. Human plasma glutathione peroxidase and symptom severity in schizophrenia. Biol. Psychiatry 1999; 45: 1512-1515.

10. Martínez-Cengotitabengoa M, Mac-Dowell KS, Leza JC et al. Cognitive impairment is related to oxidative stress and chemokine levels in first psychotic episodes. Schizophr. Res. 2012; 137: 66-72. https://doi.org/10.1016/s0006-3223(98)00184-x

11. Scottish Schizophrenia Research Group. Smoking habits and plasma lipid peroxide and vitamin E levels in nevertreated first-episode patients with schizophrenia. Br. J. Psychiatry. 2000; 176: 290293. https://doi.org/10.1192/bjp.176.3.290

12. Monin A, Fournier M, Baumann PS, Cuénod M, Do KQ. Role of redox dysregulation in white matter anomalies associated with schizophrenia. Handbook of Behavioral Neuroscience. 2016; 23:481-500. https://doi.org/10.1016/B978-0-12-800981-9.00028-6

13. Boskovic M, Vovk T, Saje S, Goricar K, Dolzan V, Plesnicar, BK, Grabnar I. Association of SOD2, GPX1, CAT, and TNF genetic polymorphisms with oxidative stress, neurochemistry, psychopathology, and extrapyramidal symptoms in schizophrenia. Neurochem Res. 2013; 38:433442. https://doi.org/10.1007/s11064-012-0937-4

14. Gysin R, Kraftsik R, Sandell J, Bovet P, Chappuis C, Conus P, et al. Impaired glutathione synthesis in schizophrenia: convergent genetic and functional evidence. Proc Natl Acad Sci U S A. 2007; 104:16621-16626. https://doi.org/10.1073/pnas.0706778104

15. Do KQ, Cabungcal JH, Frank A, Steullet P, Cuenod M. Redox dysregulation, neurodevelopment,

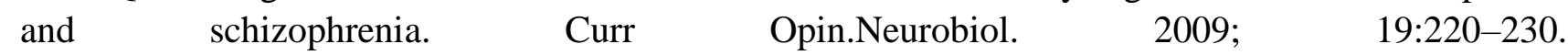
https://doi.org/10.1016/j.conb.2009.05.001

16. Madireddy S, Madireddy S. Regulation of reactive oxygen species-mediated damage in the $\begin{array}{llllll}\text { pathogenesis of } & \text { schizophrenia. } & \text { Brain }\end{array}$ https://dx.doi.org/10.3390\%2Fbrainsci10100742 
17. Antunes Neto JMF, Toyama MH, Carneiro EM, Boschero AC, Pereira-Da-Silva L, Macedo DV. Circulating leukocyte heat shock protein 70 (HSP70) and oxidative stress markers in rats after a bout of exhaustive exercise. Stress. 2006; 9: 107-115. https://doi.org/10.1080/10253890600772211

18. Shao X, Yan C, Sun D, Fu C, Tian C, Duan L, Zhu G. Association between glutathione peroxidase-1 (GPX-1) polymorphisms and schizophrenia in the chinese Han population. Neuropsychiatr Dis Treat. 2020; 16: 2297-2305. https://doi.org/10.2147/NDT.S272278

19. Ruiz-Litago F, Seco J, Echevarría E, Martínez-Cengotitabengoa M, Gil J, Irazusta J, González-Pinto AM. Adaptive response in the antioxidant defence system in the course and outcome in first-episode schizophrenia patients: a 12-months follow-up study. Psychiatry Res. 2012; 30: 218-222. https://doi.org/10.1016/j.psychres.2012.07.024

20. Kartalci S, Karabulut AB, Ozcan AC, Porgali E, Unal S. Acute and chronic effects of electroconvulsive treatment on oxidative parameters in schizophrenia patients. Prog $\begin{array}{lllll}\text { Neuropsychopharmacol } & \text { Biol } \quad \text { Psychiatry. } & 2011 ; & 135:\end{array}$ https://doi.org/10.1016/j.pnpbp.2011.05.007

21. Ciobica A, Padurariu M, Dobrin I, Stefanescu C, Dobrin R. Oxidative stress in schizophrenia focusing on the main markers. Psychiatr Danub. 2011; 23: 237-245. PMID: 21963690.

22. Sarandol A, Sarandol E, Acikgoz HE, Eker SS, Akkaya C, Dirican M. First-episode psychosis is associated with oxidative stress: Effects of short-term antipsychotic treatment. Psychiatry Clin Neurosci. 2015; 69: 699-707. https://doi.org/10.1111/pcn.12333

23. Raffa M, Atig F, Mhalla A, Kerkeni A, Mechri A. Decreased glutathione levels and impaired antioxidant enzyme activities in drug-naive first-episode schizophrenic patients. BMC Psychiatry. 2011; 11:124. https://doi.org/10.1186/1471-244x-11-124

24. Ozornina NV, Ozornin AS, Govorin N.V. Possible pathophysiological mechanisms of changes in several cytokines and in the lipid peroxidation and antioxidant defense system in first-episode $\begin{array}{lllll}\text { schizophrenia } & \text { patients. Neurochem. } & \text { J. } & 2013 ; & 7:\end{array}$ 233. https://doi.org/10.1134/S1819712413030112

25. Camkurt MA, Findiklı E, İzci F, Kurutaş EB, Tuman TC. Evaluation of malondialdehyde, superoxide dismutase and catalase activity and their diagnostic value in drug naïve, first episode, non-smoker major depression patients and healthy controls. Psychiatry Res. 2016; 238: 81-85. https://doi.org/10.1016/j.psychres.2016.01.075

26. Miljević ČD, Nikolić-Kokić A, Blagojević D, Milovanović M, Munjiza A, Jukić MM, Pešić V, Lečić-Toševski D, Spasić MB. Association between neurological soft signs and antioxidant enzyme activity in schizophrenic patients. Psychiatry Res. 2018; 269: 746-752. https://doi.org/10.1016/j.psychres.2018.09.009

27. Zhu S, Zhao L, Fan Y, Lv Q, Wu K, Lang X, Li Z, Yi Z, Geng D. Interaction between TNF- $\alpha$ and oxidative stress status in first-episode drug-naïve schizophrenia. Psychoneuroendocrinology. 2020; 114: 104595. https://doi.org/10.1016/j.psyneuen.2020.104595

28. Mukerjee S, Mahadik SP, Scheffer R, Correnti EE, Kelkar H. Impaired antioxidant defense at the onset of psychosis. Schizophr Res. 1996; 19: 19-26. https://doi.org/10.1016/0920-9964(95)00048-8

29. Wu Z, Zhang XY, Wang H, Tang W, Xia Y, Zhang F, Liu J, Fu Y, Hu J, Chen Y, Liu L, Chen DC, Xiu MH, Kosten TR, He J. Elevated plasma superoxide dismutase in first-episode and drug naive patients with schizophrenia: inverse association with positive symptoms. Prog $\begin{array}{llllll}\text { Neuropsychopharmacol } & \text { Biol } & \text { Psychiatry. } & 2012 ; & 36\end{array}$ https://doi.org/10.1016/j.pnpbp.2011.08.018

30. Bai ZL, Li XS, Chen GY, Du Y, Wei ZX, Chen X, Zheng GE, Deng W, Cheng Y. Serum oxidative stress marker levels in unmedicated and medicated patients with schizophrenia. J Mol Neurosci. 2018; 66: 428-436. https://doi.org/10.1007/s12031-018-1165-4

31. Jordan W, Dobrowolny H, Bahn S, Bernstein HG, Brigadski T, Frodl T, Isermann B, Lessmann V, Pilz J, Rodenbeck A, Schiltz K, Schwedhelm E, Tumani H, Wiltfang J, Guest PC, Steiner J. Oxidative stress in drug-naïve first episode patients with schizophrenia and major depression: effects 
of disease acuity and potential confounders. Eur Arch Psychiatry Clin Neurosci. 2018; 268: 129-143. https://doi.org/10.1007/s00406-016-0749-7

32. Lang X, Wang DM, Du XD, Jia QF, Chen DC, Xiu M, Wang L, Zhang X. Elevated activity of plasma superoxide dismutase in never-treated first-episode schizophrenia patients: Associated with $\begin{array}{lllll}\text { depressive } & \text { symptoms. } & \text { Schizophr } & \text { Res. 2020; }\end{array}$ https://doi.org/10.1016/j.schres.2020.05.032

33. Xiu MH, Li Z, Chen DC, Chen S, Curbo ME, Wu HE, Tong YS, Tan SP, Zhang WY. Interrelationships between BDNF, superoxide dismutase, and cognitive impairment in drug-naive first-episode patients with schizophrenia. Schizophrenia Bulletin. 2020; 46: 1498-1510. https://doi.org/10.1093/schbul/sbaa062

34. Wang DM, Chen DC, Wang L, Zhang XY. Sex differences in the association between symptoms and superoxide dismutase in patients with never-treated first-episode schizophrenia. World J Biol Psychiatry. 2021; 22: 325-334. https://doi.org/10.1080/15622975.2020.1805510

35. Reyazuddin M, Azmi SA, Islam N, Rizvi A. Oxidative stress and level of antioxidant enzymes in drug-naive schizophrenics. Indian J Psychiatry. 2014; 56: 344-349. https://doi.org/10.4103/00195545.146516

36. Langbein K, Hesse J, Gussew A, Milleit B, Lavoie S, Amminger GP, Gaser C, Wagner G, Reichenbach JR, Hipler UC, Winter D, Smesny S. Disturbed glutathione antioxidative defense is associated with structural brain changes in neuroleptic-naïve first-episode psychosis patients. Prostaglandins Leukot Essent Fatty Acids. 2018; 136: 103-110. https://doi.org/10.1016/j.plefa.2017.10.005

37. Reyes-Madrigal F, León-Ortiz P, Mao X, Mora-Durán R, Shungu DC, de la Fuente-Sandoval C. Striatal Glutathione in First-episode Psychosis Patients Measured In Vivo with Proton Magnetic Resonance Spectroscopy. Arch Med Res. 2019; 50: 207-213. https://doi.org/10.1016/j.arcmed.2019.08.003

38. Mahadik SP, Mukherjee S, Scheffer R, Correnti EE, Mahadik JS. Elevated plasma lipid peroxides at the onset of nonaffective psychosis. Biol Psychiatry. 1998; 43: 674-679. https://doi.org/10.1016/s0006-3223(97)00282-5

39. Khan MM, Evans DR, Gunna V, Scheffer RE, Parikh VV, Mahadik SP. Reduced erythrocyte membrane essential fatty acids and increased lipid peroxides in schizophrenia at the never-medicated first-episode of psychosis and after years of treatment with antipsychotics. Schizophr Res. 2002; 58: 1-10. https://doi.org/10.1016/s0920-9964(01)00334-6

40. Noto C, Ota VK, Gadelha A, Noto MN, Barbosa DS, Bonifácio KL, Nunes SO, Cordeiro Q, Belangero SI, Bressan RA, Maes M, Brietzke E. Oxidative stress in drug naïve first episode psychosis and antioxidant effects of risperidone. J Psychiatr Res. 2015; 68: 210-216. https://doi.org/10.1016/j.jpsychires.2015.07.003

41. Hozo SP, Djulbegovic B, Hozo I. Estimating the mean and variance from the median, range, and the size of a sample. BMC Med Res Methodol. 2005; 5: 13. https://doi.org/10.1186/1471-2288-5-13

42. Maher CG, Sherrington C, Herbert RD, Moseley AM, Elkins M. Reliability of the PEDro scale for rating quality of randomized controlled trials. Phys Ther. 2003 Aug;83(8):713-21. https://doi.org/10.1093/PTJ\%2F83.8.713

43. Murray AJ, Rogers JC, Katshu MZUH, Liddle PF, Upthegrove R. Oxidative stress and the pathophysiology and symptom profile of schizophrenia spectrum disorders. Front Psychiatry. 2021; 12: 703452. https://dx.doi.org/10.3389\%2Ffpsyt.2021.703452

44. Huang D, Liu S. Oxidative stress and schizophrenia. Journal of Psychiatry and Brain Science. 2017; 2: 1-4. https://doi.org/10.20900/jpbs.20170006

45. Fraguas D, Díaz-Caneja CM, Rodríguez-Quiroga A, Arango C. Oxidative Stress and Inflammation in Early Onset First Episode Psychosis: A Systematic Review and Meta-Analysis. Int J Neuropsychopharmacol. 2017; 20: 435-444. https://doi.org/10.1093/ijnp/pyx015 
46. Zhang H, Wang Y, Hu Y, Zhu Y, Zhang T, Wang J, Ma K, Shi C, Yu X, Li C. Meta-analysis of cognitive function in Chinese first-episode schizophrenia: MATRICS Consensus Cognitive Battery (MCCB) profile of impairment. Gen Psychiatr. 2019; 32: e100043. https://doi.org/10.1136/gpsych2018-100043

47. Padurariu M, Ciobica A, Hritcu L, Stoica B, Bild W, Stefanescu C. Changes of some oxidative stress markers in the serum of patients with mild cognitive impairment and Alzheimer's disease. Neurosci Lett. 2010; 469:6-10. https://doi.org/10.1016/j.neulet.2009.11.033

48. Baldeiras I, Santana I, Proença MT. Peripheral oxidative damage in mild cognitive impairment and mild Alzheimer's disease. J Alzheimers Dis. 2008; 15:117-28. https://doi.org/10.3233/jad-200815110

49. Greilberger J, Koidl C, Greilberger M. Malondialdehyde, carbonyl proteins and albumin-disulphide as useful oxidative markers in mild cognitive impairment and Alzheimer's disease. Free Radic Res. 2008; 42: 633-8. https://doi.org/10.1080/10715760802255764

50. Kunz M, Gama CS, Andreazza AC. Elevated serum superoxide dismutase and thiobarbituric acid reactive substances in different phases of bipolar disorder and in schizophrenia. Prog $\begin{array}{llllll}\text { Neuropsychopharmacol } & \text { Biol } & \text { Psychiatry. } & 2008 ; & 32: & \text { 1677-81. }\end{array}$ https://doi.org/10.1016/j.pnpbp.2008.07.001

51. Ballesteros A, Summerfelt A, Du X, Jiang P, Chiappelli J, Tagamets M, O'Donnell P, Kochunov P, Hong LE. Electrophysiological intermediate biomarkers for oxidative stress in schizophrenia. Clin Neurophysiol. 2013; 124: 2209-2215. https://doi.org/10.1016/j.clinph.2013.05.021

52. Zeni-Graiff M, Rios AC, Maurya PK, Rizzo LB, Sethi S, Yamagata AS, Mansur RB, Pan PM, Asevedo E, Cunha GR, Zugman A, Bressan RA, Gadelha A, Brietzke E. Peripheral levels of superoxide dismutase and glutathione peroxidase in youths in ultra-high risk for psychosis: a pilot study. CNS Spectr. 2019; 24: 333-337. https://doi.org/10.1017/s1092852917000803

53. Fendri C, Mechri A, Khiari G, Othman A, Kerkeni A, Gaha L. Oxidative stress involvement in schizophrenia pathophysiology: a review. Encéphale. 2006; 32: 244-252. https://doi.org/10.1016/s0013-7006(06)76151-6

54. Mahadik SP, Mukherjee S. Free radical pathology and antioxidant defense in schizophrenia: a review. Schizophr Res. 1996; 19: 1-7. https://doi.org/10.1016/0920-9964(95)00049-6

55. Ng F, Berk M, Dean O, Bush AI. Oxidative stress in psychiatric disorders: evidence base and therapeutic implications. Int J Neuropsychopharmacol. 2008; 11: 851-876. https://doi.org/10.1017/s1461145707008401

56. Reddy R, Keshavan M, Yao JK. Reduced plasma antioxidants in first-episode patients with schizophrenia. Schizophr Res. 2003; 62: 205-212. https://doi.org/10.1016/s0920-9964(02)00407-3

57. Yao JK, Leonard S, Reddy RD. Increased nitric oxide radicals in postmortem brain from patients $\begin{array}{lllll}\text { with } & \text { schizophrenia. } & \text { Schizophr } & \text { Bull. } & \text { 2004; }\end{array}$ https://doi.org/10.1093/oxfordjournals.schbul.a007142 